\title{
Correlated optical, X-ray, and $\gamma$-ray flaring activity seen with INTEGRAL during the 2015 outburst of V404 Cygni ${ }^{\star}$
}

\author{
J. Rodriguez ${ }^{1}$, M. Cadolle Bel ${ }^{2}$, J. Alfonso-Garzón ${ }^{3}$, T. Siegert ${ }^{4}$, X.-L. Zhang ${ }^{4}$, V. Grinberg ${ }^{5}$, V. Savchenko ${ }^{6}$, \\ J. A. Tomsick ${ }^{7}$, J. Chenevez ${ }^{8}$, M. Clavel ${ }^{1}$, S. Corbel ${ }^{1}$, R. Diehl ${ }^{4}$, A. Domingo ${ }^{3}$, C. Gouiffès ${ }^{1}$, J. Greiner ${ }^{4}$, \\ M. G. H. Krause ${ }^{9,4}$, P. Laurent ${ }^{6}$, A. Loh ${ }^{1}$, S. Markoff ${ }^{10}$, J. M. Mas-Hesse ${ }^{3}$, \\ J. C. A. Miller-Jones ${ }^{11}$, D. M. Russell ${ }^{12}$, and J. Wilms ${ }^{13}$
}

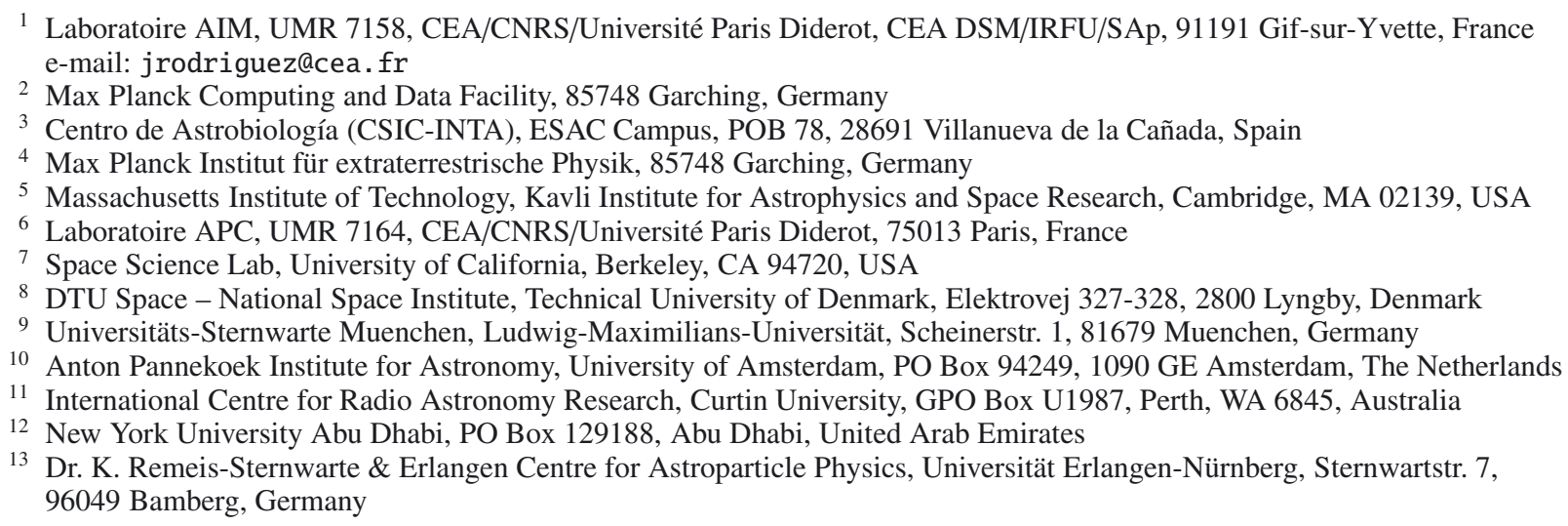

Received 23 July 2015 / Accepted 31 August 2015

\section{ABSTRACT}

\begin{abstract}
After 25 years of quiescence, the microquasar V404 Cyg entered a new period of activity in June 2015. This X-ray source is known to undergo extremely bright and variable outbursts seen at all wavelengths. It is therefore an object of prime interest to understand the accretion-ejection connections. These can, however, only be probed through simultaneous observations at several wavelengths. We made use of the INTEGRAL instruments to obtain long, almost uninterrupted observations from 2015 June 20, 15:50 UTC to June 25, 4:05 UTC, from the optical $V$ band up to the soft $\gamma$-rays. V404 Cyg was extremely variable in all bands, with the detection of 18 flares with fluxes exceeding $6 \mathrm{Crab}(20-40 \mathrm{keV})$ within three days. The flare recurrence can be as short as $\sim 20$ min from peak to peak. A model-independent analysis shows that the $>6 \mathrm{Crab}$ flares have a hard spectrum. A simple $10-400 \mathrm{keV}$ spectral analysis of the off-flare and flare periods shows that the variation in intensity is likely to be only due to variations of a cut-off power-law component. The optical flares seem to be at least of two different types: one occurring in simultaneity with the X-ray flares, the other showing a delay greater than $10 \mathrm{~min}$. The former could be associated with X-ray reprocessing by either an accretion disk or the companion star. We suggest that the latter are associated with plasma ejections that have also been seen in radio.
\end{abstract}

Key words. accretion, accretion disks - X-rays: binaries - radio continuum: stars - stars: black holes - stars: individual: V404 Cygni

\section{Introduction}

V404 Cyg (hereafter V404) is a low-mass X-ray binary (LMXB) consisting of a black hole $(\mathrm{BH})$ with mass estimates ranging from $\sim 9$ to $15 M_{\odot}$, and a $0.7_{-0.2}^{+0.3} M_{\odot} \mathrm{K} 3$ III companion (Casares \& Charles 1994; Shahbaz et al. 1994; Khargharia et al. 2010), located at a parallax distance $2.39 \pm 0.14 \mathrm{kpc}$ (Miller-Jones et al. 2009). The inclination of the binary's rotational axis is $67^{\circ+3}$ (Shahbaz et al. 1994; Khargharia et al. 2010), the orbital period $6.5 \mathrm{~d}$ (Casares et al. 1992). This transient underwent three periods of outbursts during the twentieth century (Richter 1989), the last, in May 1989, leading to its discovery as an X-ray transient by the Ginga satellite (as GS 2023+338, Makino et al. 1989). V404 showed bright X-ray flares on short time-scales (e.g. Makino et al. 1989; Terada et al. 1994), which makes it an excellent source to study the connections between the accretion

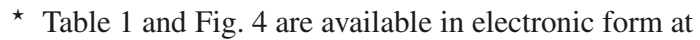
http: //wwW . aanda.org and ejection phenomena, which are the probable origin of this behaviour. V404 is one of the closest stellar mass BHs, making it a rare case where quiescence can be studied in detail. Variable remnant activity, attributed to a compact jet, was detected from radio to hard X-rays (e.g. Hynes et al. 2004; Xie et al. 2014). V404 is one of the few sources that defines the radio/X-ray correlation over a wide range of luminosities, down into quiescence (Corbel et al. 2008). The good knowledge of the quiescent state makes understanding new outburst observations paramount as they allow the mechanisms responsible for the increased activity to be probed.

On 2015 June 15 (MJD 57 188), V404 went into outburst again. It was first detected by Swift (BAT and XRT) (Barthelmy et al. 2015) and then with MAXI and INTEGRAL (Negoro et al. 2015; Kuulkers et al. 2015). These early alerts triggered follow-up observations at all wavelengths. Preliminary results all report the detection of the source, variations of specific spectral features, and an extreme flaring activity at all wavelengths 

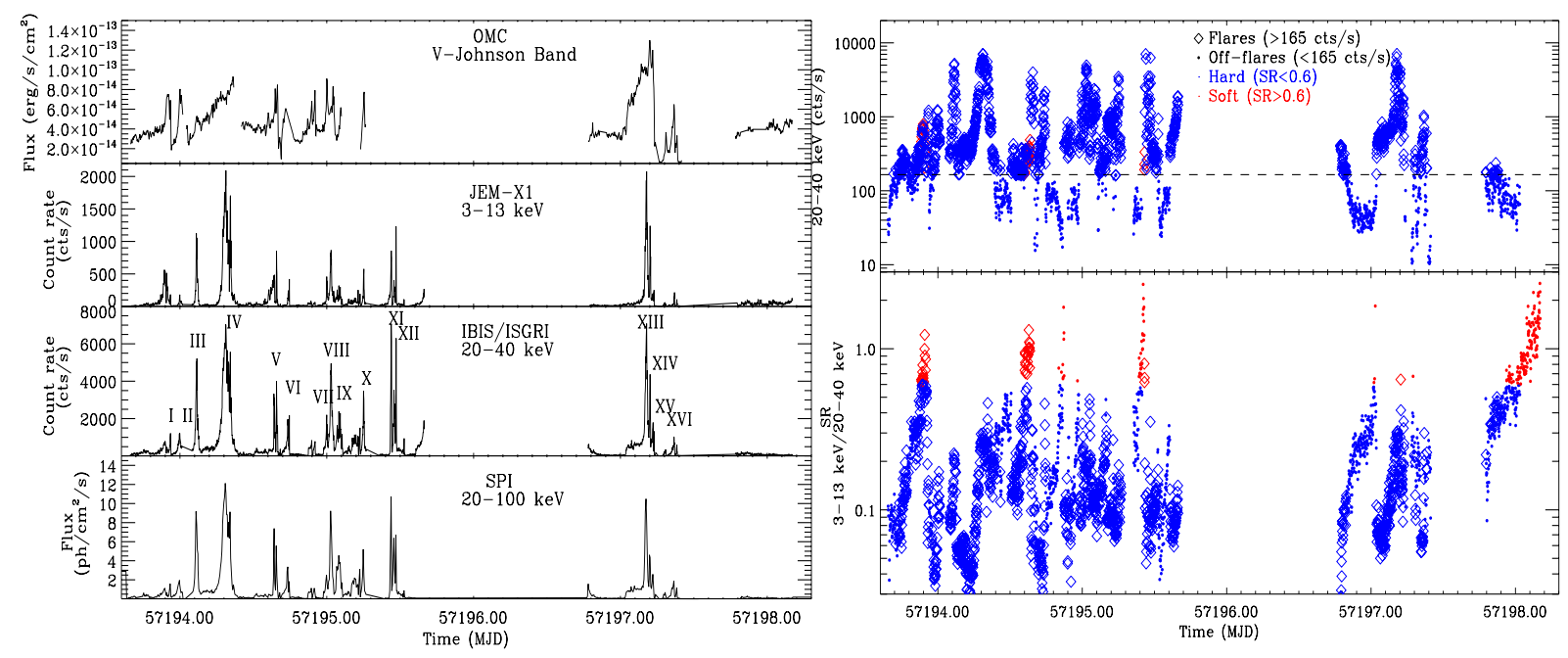

Fig. 1. Left: INTEGRAL LCs of V404 in four spectral domains (a larger version of the plot including all energy ranges is available as Fig. 4). Right: 20-40 keV count rate (top), and 3-13/20-40 keV softness ratio (SR, bottom). The dashed horizontal line corresponds to $1 \mathrm{Crab}$ (20-40 keV). MJD 57193 is 2015 June 20.

(e.g. Mooley et al. 2015; Munoz-Darias et al. 2015; Ferrigno et al. 2015; Motta et al. 2015; Tetarenko et al. 2015a,b; Tsubono et al. 2015). We triggered our INTEGRAL ToO program to obtain quasi-continuous coverage in $\mathrm{X} / \gamma$-rays and in the optical $V$ band. The first detection of multiple flares exceeding $30 \mathrm{Crab}$ in $20-40 \mathrm{keV}$ and possible correlated flaring in the $V$ band were reported in Rodriguez et al. (2015a) and Domingo et al. (2015).

Our observations caught the source during the most intense and variable phase of this new outburst. Here, we first give the details of the observations and data reduction (Sect. 2). We then focus on the flaring behaviour at high energies (Sect. 3), which we compare to the activity in the optical $V$ band (Sect. 4). A preliminary phenomenological spectral characterization of different intensity intervals is presented in Sect. 5. We discuss our results and compare V404 to other microquasars in Sect. 6.

\section{Observations and data reduction}

Our ToO program (Fig. 1) covered MJD 57 193.66-57 198.17 (2015-06-20 15:50 UTC to 2015-06-25 4:05 UTC), that is, INTEGRAL revolutions 1555 (continuous coverage) and 1556 (two periods). The data of all the INTEGRAL instruments (see Winkler et al. 2003, and references therein for all instrumental details) were reduced with the Off line Scientific Analysis (OSA) v10.1 software suite, with the latest calibration files available at the time of writing.

Images and $100 \mathrm{~s}$ binned light curves (LC) from the Joint European X-ray Monitors (JEM-X) and the Imager on Board the INTEGRAL Satellite (IBIS) were produced in two bands (3-13, and $13-30 \mathrm{keV})$ for JEM-X unit 1, and in four bands (20-40, 40-80, 80-150, and 150-300 keV) for the IBIS Soft GammaRay Imager (ISGRI).

The event data of the Spectrometer on INTEGRAL (SPI) were fitted with models for the celestial sources and instrumental background following standard reduction processes. The 20$100 \mathrm{keV} \mathrm{LC}$ of V404 as well as the other sources in the field were obtained in bins of $400 \mathrm{~s}$. Background models were built based on the pre-flaring data of a representative empty sky region, adjusting the normalization coefficient per hour (see, e.g., Strong et al. 2005, for a more general description of the method).

As source intensity and hardness vary strongly on short timescales, we extracted luminosity/hardness dependent JEM-X, ISGRI, and SPI spectra over specific time intervals of clean data. The spectra from the same time intervals were jointly fitted within XSPEC v12.8.2. Since the instruments' responses are possibly different for the high intensities observed, only phenomenological spectral fits are presented, and the fit results should be viewed with some caution.

The INTEGRAL/Optical Monitoring Camera (OMC) fluxes and magnitudes were derived from a photometric aperture of $3 \times 3$ pixels $\left(1\right.$ pix. $\left.=17.504^{\prime \prime}\right)$, slightly circularized, that is, removing one quarter pixel from each corner (standard output from OSA). The photometric aperture was centred on the source coordinates (default centroid algorithm) and did not include any significant contribution from other objects. We removed measurements with a severe problem flag, and, to restrict the noise, only measurements of 50 and $200 \mathrm{~s}$ duration were considered.

\section{Model-independent description of the flaring}

Multi-wavelength LCs of V404 from the $V$ band up to $\gamma$-rays are highly structured with several large flares separated by calmer periods seen in all bands (Fig. 1, and see also Fig. 4 for a plot with all energy ranges). In the following, count rates (CR) are given in the ISGRI 20-40 keV range. When the source CR increased above $\sim 150-200 \mathrm{cts} / \mathrm{s}$, an intense X-ray flare systematically followed. In the following, we thus set $1 \mathrm{Crab}^{1}$ as the typical limit between the off-flare and flaring intervals. We identified 18 main events, that is, peaks that reached at least $6 \mathrm{Crab}$ (labelled with Roman numerals ${ }^{2}$ in Fig. 1, their main characteristics are given in Table 1), with 11 exceeding 20 Crab during our observations. Flares IV, XI, and XIII are the brightest we observed, reaching $43 \mathrm{Crab}$. The flares occurred isolated (e.g. III, IV, VI) as well as in groups with peak-to-peak intervals as short as $22 \mathrm{~min}(\mathrm{Va}, \mathrm{Vb})$ The flares lasted $0.4-2.4 \mathrm{~h}$, except for peaks IV and XIII. The former shows a rather broad profile and has multiple peaks. This event lasted $4.8 \mathrm{~h}$ in total and is the longest flare of our observation. The latter reached about $40 \mathrm{Crab}$. The peak itself lasted about $1.5 \mathrm{~h}$, but was preceded by a $\sim 3 \mathrm{~h}$ long, $3 \mathrm{Crab}$ plateau seen only above $13 \mathrm{keV}$. It was followed by flares $\mathrm{XIV}$ and $\mathrm{XV}$, which show decreasing peak values.

\footnotetext{
1 The ISGRI/20-40 keV CR of the Crab is $165 \mathrm{cts} / \mathrm{s} \Leftrightarrow F_{20-40 \mathrm{keV}}=$ $8 \times 10^{-9} \mathrm{erg} / \mathrm{cm}^{2} / \mathrm{s}$ for a power-law spectrum with $\Gamma=2.1$ and a normalization of $10 \mathrm{ph} / \mathrm{cm}^{-2} / \mathrm{s}$ at $1 \mathrm{keV}$.

$2 \mathrm{~V}$ and XII contain two distinct events that are hardly distinguishable in Fig. 1. They appear under the same label in Fig. 1 (to keep it clear), and are named with a/b sub-labels in the text and Table 1.
} 

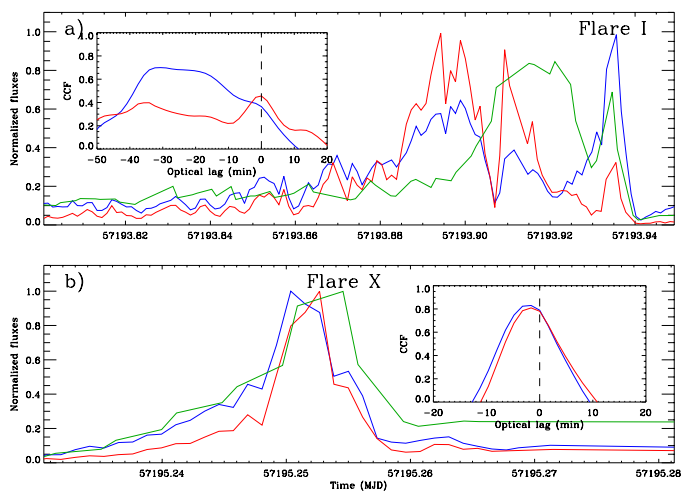

Fig. 2. $V$-band (green), 3-13 keV (red), and 20-40 keV (blue) LCs around flares $\mathrm{I} \mathbf{a})$ and $\mathrm{X} \mathbf{b}$ ). The inserts show the cross-correlation functions of the 3-13 keV (red) and 20-40 keV (blue) vs. the optical LCs over the same time intervals as the LCs. The dashed vertical lines represent the 0 lag level.

The 3-13/20-40 keV softness ratio (SR, Fig. 1, right) shows that the strong variability of the source is associated with variations of SR from $\sim 0.03$ to $\sim 1.3$ corresponding to $\Gamma=0.1-2.5$ in simulated JEM-X/ISGRI power-law spectra. SR $\sim 0.6$ corresponds to $\Gamma=2.0$. Strong spectral variations are visible in the off-flare intervals (Fig. 1, right). All the flares are hard, and all have $\mathrm{SR}<0.4(\Leftrightarrow \Gamma<1.8)$.

\section{Optical vs. X-ray behaviour}

The comparison of the optical (OMC) and X-ray (JEM-X1 and ISGRI) LCs shows a non-trivial relationship. Significant flaring activity is evident in the $V$ band LC (Fig. 1, left), with at least 12 clear flares. The optical flare typically lasted $0.24-2.5 \mathrm{~h}$. While some events occurred in simultaneity with X-ray flares, the optical emission was delayed with respect to the X-rays in other cases. Figure 2 shows typical examples of these different behaviours. The cross-correlation function (ccf) between the X-ray and optical emission confirms the absence of lags for some of the flares (e.g. flare I, which causes the peak at 0 in the ccf of Fig. 2a), and delayed optical emission from $1.5 \mathrm{~min}$ to 20-30 min is seen in others. The ccf of Fig. 2b shows an example of a $\sim 3$ min lag, while the ccf of Fig. 2 a (in addition to showing the simultaneity of peak I and its optical counterpart) shows lag at 20-30 min, representing the delay between the small X-ray flares preceding peak I (around MJD 53 193.9) and the subsequent optical flare (around MJD 53 193.92). Because of the time resolution of $\mathrm{OMC}$, however, lags shorter than 1 min cannot be measured with our data, and additional lags of the order of seconds are not excluded.

While most of the flares show a fast rise similar to the flares observed in X-rays, the two optical events occurring close to X-ray peaks IV and XIII seem to be exceptional. All other flares show fast rises $(\lesssim 1 \mathrm{~h})$, but these two events have slower rises (about 10 and $4 \mathrm{~h}$, respectively), and are both coincident with hard plateaus that precede the X-ray peaks.

\section{Spectral analysis}

We accumulated spectra from the brightest flares $(\mathrm{CR}>1000 \mathrm{cts} / \mathrm{s})$ and the off-flare intervals $(\mathrm{CR}<165 \mathrm{cts} / \mathrm{s})$. In the latter case we also only retained the hard intervals $(\mathrm{SR}<0.6)$ in order to exclude the softening visible after MJD 57197.9 (Fig. 1). The resulting $v-f_{v}$ spectra are plotted in Fig. 3.

The off-flare spectrum is well fitted $\left(\chi_{v}^{2}=1.2,66\right.$ degrees of freedom, d.o.f.) by a model consisting of a power law with a high-energy cut-off dominating at $10-100 \mathrm{keV}$ plus an additional power law dominating above $100 \mathrm{keV}$. The former component

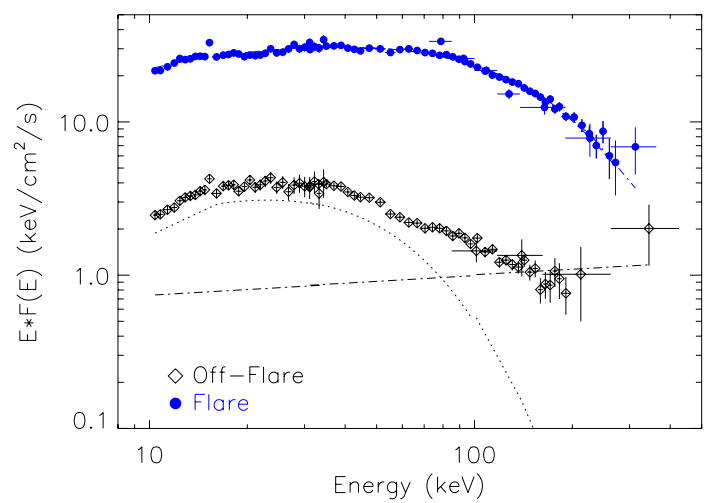

Fig. 3. $v-f_{v}$ spectra obtained from the fits to the off-flare and flare intervals. The lines represent the spectral components used for the fits.

has $\Gamma=1.0_{-0.4}^{+0.3}, E_{\text {cut }}=16_{-2}^{+4} \mathrm{keV}, E_{\text {fold }}=23 \pm 5 \mathrm{keV}$, the latter has $\Gamma=1.9_{-0.3}^{+0.2}$ ( $\Gamma$ is the photon index defined as $N(E) \propto E^{-\Gamma}$.) Normalization constants were included to account for potential cross-calibration issues or differences in the effective exposures (deadtime corrections, telemetry drop out). When set to 1 for ISGRI, we obtain $\sim 1.9$ for SPI and $\sim 0.6$ for JEM-X1. The 20 $400 \mathrm{keV}$ (ISGRI) flux is $\sim 10^{-8} \mathrm{erg} / \mathrm{cm}^{2} / \mathrm{s}$, and the above model leads to an extrapolated $0.1-10^{5} \mathrm{keV}$ flux $\sim 3.8 \times 10^{-8} \mathrm{erg} / \mathrm{cm}^{2} / \mathrm{s}$, that is, about $2 \% L_{\text {Edd }}$ for a $9 M_{\odot} \mathrm{BH}$.

The flare spectrum is well represented $\left(\chi_{v}^{2} \sim 0.9,78\right.$ d.o.f.) by a single cut-off power law with $\Gamma=1.54 \pm 0.06$, $E_{\text {cut }}=14.0_{-3.3}^{+2.8} \mathrm{keV}, E_{\text {fold }}=87_{-5}^{+4} \mathrm{keV}$. An extra power-law component is not statistically required according to an F-test. The normalization constants are both close to 1.1 The $20-400 \mathrm{keV}$ (ISGRI) flux is $\sim 10^{-7} \mathrm{erg} / \mathrm{cm}^{2} / \mathrm{s}$, which leads to an extrapolated $0.1-10^{5} \mathrm{keV}$ flux $\sim 310^{-7} \mathrm{erg} / \mathrm{cm}^{2} / \mathrm{s}$, or about $20 \% L_{\text {Edd }}$ for a $9 M_{\odot} \mathrm{BH}$.

\section{Discussion}

Over the four days covered by our INTEGRAL ToO, V404 showed a high level of emission with sporadic flares with a maximum 20-40 keV dynamical range of 940 (flare XVI). During its flares, V404 became the brightest X-ray object in the sky. In the hard off-flare state the spectral analysis shows two spectral components: a cut-off power law typically attributed to thermal Comptonization and an extra power law at energies beyond $100 \mathrm{keV}$. Hard tails have now been seen in a large number of systems (e.g. GRS 1915+105, Swift J1753.5-0127, GX 339-4, or Cyg X-1, Rodriguez et al. 2008b, 2015b; Tomsick et al. 2015; Joinet et al. 2007), and their origin is still highly debated, although a compact jet origin is favoured in the case of Cyg X-1 (e.g. Russell \& Shahbaz 2014; Rodriguez et al. 2015b). It is interesting that the flaring activity seems primarily due only to spectral variations of the cut-off power law. We estimate an integrated luminosity $L \sim 0.2 L_{\text {Edd }}$ for the $>6$ Crab flares. This is a lower limit to the maximum luminosity reached at the peak of the brightest flares. First because we averaged the data, without isolating the brightest portions of the flares. Moreover, we did not consider all the contributions below $10 \mathrm{keV}$ (disk, jet) that can provide a significant fraction of the bolometric luminosity. Assuming a simple scaling between the CR and $L_{\text {Edd }}$ with a constant shape for the variable component between the offflare hard state and the flares, we conclude that all peaks with $\mathrm{CR} \gtrsim 3000 \mathrm{cts} / \mathrm{s}(\sim 18 \mathrm{Crab})$ (flares III, IV, Va,b, VIII, X, XI, XIIa,b, XIII and XIV, Fig. 1$)$ reached $\sim L_{\text {Edd }}$.

The optical activity is also highly variable and shows flares (Figs. 1 and 2). Some optical flares occur in conjunction with the X-rays, while other activity periods show delays (Fig. 2). The first specific length scale of this system is the separation 
between the $\mathrm{BH}$ and the companion: using the system parameters from Sect. 1 and a $9 M_{\odot} \mathrm{BH}$, we estimate $\sim 2.2 \times 10^{12} \mathrm{~cm}$ or $\sim 75$ light seconds. Hence, when no delay between the optical and the X-rays is observed (e.g. flare I in Fig. 2a), the mechanism producing the optical emission could be related to X-ray reprocessing, either by an accretion disk or by the companion. The maximum delays expected would be around $60 \mathrm{~s}$ (outer disk), and $\lesssim 150 \mathrm{~s}$ (companion located at superior conjunction).

Optical lags $\gtrsim 10$ min could be related to variable jet properties, either as their intrinsic synchrotron emission, or from their interaction with the surrounding medium. Radio and millimeter $(\mathrm{mm})$ flaring activity ascribed to discrete ejections has been reported during this outburst (e.g. Sivakoff et al. 2015; Mooley et al. 2015; Tetarenko et al. 2015b). Delays between $\mathrm{X}$-rays and longer wavelengths are expected in the case of adiabatically expanding ejecta (van der Laan 1966; Mirabel et al. 1998), and Tetarenko et al. (2015b) reported a mm peak on MJD 57 195.548, about $26 \mathrm{~min}$ after the MJD 57195.53 X-ray event (a $<6$ Crab flare that occurred slightly after flare XII). Interestingly, this delay also corresponds to the time scale of the $\mathrm{mm}$ flare increase (Tetarenko et al. 2015b), which renders the $\mathrm{mm}$ compatible with being causally related to the X-ray flare. As in other well-known sources, e.g. XTE J1550-564, GRO J1655-40, GRS 1915+105 (Fender 2006), discrete ejections may be causally connected to the X-ray activity in V404.

V404 reached maximum absolute peak intensities that are rather usual during outbursts of microquasars. If V404 was at $10 \mathrm{kpc}$, the maximum peak intensity would have been $2.5 \mathrm{Crab}$, a value similar to the aforementioned other microquasars during their brightest outburst(s) (e.g. Remillard \& McClintock 2006). Short recurrent (multi-wavelength) flares have been seen only in GRS 1915+105 (e.g. Greiner et al. 1996), however, and in V404 the flaring activity similarly recurs on time scales as short as $22 \mathrm{~min}$. Some of the V404 optical flares lag $\gtrsim 20 \mathrm{~min}$ behind the X-rays (Fig. 2a), and similar lags are also seen at mm-radio wavelengths. This may resemble the correlated $\mathrm{X}$-ray/infrared/radio oscillations also referred to as 30-min cycles of GRS 1915+105 (e.g. Fender \& Pooley 1998; Mirabel et al. 1998; Rodriguez et al. 2008a). In GRS 1915+105, however, these events are associated with hard X-ray dips preceding the flares and a clear softening at the X-ray peak, marking the disappearance of the Comptonization component (Rodriguez et al. 2008b). V404, in contrast, remains hard even in the flaring states (Fig. 1), indicating that a different mechanism is responsible for the X-ray flaring (similar results were obtained from the 1989 outburst, e.g. Życki et al. 1999). One tempting possibility would be that the high-energy flares are direct boosted emission from a jet (blazar-like configuration). This would imply a jet axis not perpendicular to the orbital plane. Misaligned jets have been seen in GRO J1655-40 and V4641 Sgr (Maccarone 2002, and references therein). In the former, a rather modest Lorentz factor $\gamma \sim 2.5$ implies weak relativistic boosting (Hjellming \& Rupen 1995). In the latter, $\gamma$ ranges from 10 up to 17 , and the angle between the jet axis and the orbital plane normal is as high as $50^{\circ}$ (Maccarone 2002, and references therein). Significant Doppler boosting is expected in this case.

Życki et al. (1999) argued that the spectral and intensity variability seen with Ginga in 1989 could be due to the evolution of a heavily absorbing medium. However, even with $N_{\mathrm{H}} \gtrsim 10^{24} \mathrm{~cm}^{-2}$ (Życki et al. 1999), the activity above $20 \mathrm{keV}$ is not affected by absorption, and hence the absorber alone cannot be responsible for the strong variability we observe. The high-energy flares could be due to the shock of the relativistic jets with the dense ambient medium. Then optically thin synchrotron emission would be expected at X-ray energies, while our analysis favours thermal Comptonization. More simultaneous multi-wavelength observations will help distinguish these different possibilities.

Acknowledgements. We warmly thank the referee for the useful report that helped us to improve the quality of this paper. We also thank the INTEGRAL teams and planners for their prompt reaction and the scheduling of these observations. J.R., M.C., S.C. acknowledge funding support from the French Research National Agency: CHAOS project ANR-12-BS05-0009 (http:// www.chaos-project.fr), and from the UnivEarthS Labex program of Sorbonne Paris Cité (ANR-10-LABX-0023 and ANR-11-IDEX-0005-02). XLZ acknowledges funding through DLR 50 OG 1101. M.G.H.K. was supported by the Deutsche Forschungsgemeinschaft under DFG project number PR 569/10-1 in the context of the Priority Program 1573 Physics of the Interstellar Medium. This work was supported by NASA through the Smithsonian Astrophysical Observatory (SAO) contract SV3-73016 for the Chandra X-Ray Center and Science Instruments. R.D. and X.L.Z. acknowledge support through the Deutsches Zentrum für Luft- und Raumfahrt e.V. (DLR) 50 OG 1101. O.M.C. activities are supported by Spanish MINECO grant ESP2014-59789-P. This study is based on observations made with INTEGRAL, an ESA project with instruments and science data centre funded by ESA member states, Poland and with the participation of Russia and the USA.

\section{References}

Barthelmy, S. D., D'Ai, A., D'Avanzo, P., et al. 2015, GRB Coordinates Network, 17929, 1

Casares, J., \& Charles, P. A. 1994, in The Evolution of X-ray Binariese, eds. S. Holt, \& C. S. Day, AIP Conf. Ser., 308, 107

Casares, J., Charles, P. A., \& Naylor, T. 1992, Nature, 355, 614

Corbel, S., Koerding, E., \& Kaaret, P. 2008, MNRAS, 389, 1697

Domingo, A., Alfonso-Garzon, J., Mas-Hesse, J. M., Rodriguez, J., \& Bel, M. C. 2015, ATel, 7717,1

Fender, R. 2006, in Compact stellar X-ray sources (Cambridge, UK: Cambridge University Press), 381

Fender, R. P., \& Pooley, G. G. 1998, MNRAS, 300, 573

Ferrigno, C., Fotopoulou, S., Domingo, A., et al. 2015, ATel, 7662, 1

Greiner, J., Morgan, E. H., \& Remillard, R. A. 1996, ApJ, 473, L107

Hjellming, R. M., \& Rupen, M. P. 1995, Nature, 375, 464

Hynes, R. I., Charles, P. A., Garcia, M. R., et al. 2004, ApJ, 611, L125

Joinet, A., Jourdain, E., Malzac, J., et al. 2007, ApJ, 657, 400

Khargharia, J., Froning, C. S., \& Robinson, E. L. 2010, ApJ, 716, 1105

Kuulkers, E., Motta, S., Kajava, J., et al. 2015, ATel, 7647, 1

Maccarone, T. J. 2002, MNRAS, 336, 1371

Makino, F., Wagner, R. M., Starrfield, S., et al. 1989, IAU Circ., 4786, 1

Miller-Jones, J. C. A., Jonker, P. G., Dhawan, V., et al. 2009, ApJ, 706, L230

Mirabel, I. F., Dhawan, V., Chaty, S., et al. 1998, A\&A, 330, L9

Mooley, K., Fender, R., Anderson, G., et al. 2015, ATel, 7658, 1

Motta, S., Beardmore, A., Oates, S., et al. 2015, ATel, 7666, 1

Munoz-Darias, T., Sanchez, D. M., Casares, J., et al. 2015, ATel, 7659, 1

Negoro, H., Matsumitsu, T., Mihara, T., et al. 2015 ATel, 7646, 1

Remillard, R. A., \& McClintock, J. E. 2006, ARA\&A, 44, 49

Richter, G. A. 1989, IBVS, 3362, 1

Rodriguez, J., Hannikainen, D. C., Shaw, S. E., et al. 2008a, ApJ, 675, 1436 Rodriguez, J., Shaw, S. E., Hannikainen, D. C., et al. 2008b, ApJ, 675, 1449 Rodriguez, J., Ferrigno, C., Cadolle Bel, M., et al. 2015a, ATel, 7702, 1

Rodriguez, J., Grinberg, V., Laurent, P., et al. 2015b, ApJ, 807, 17

Russell, D. M., \& Shahbaz, T. 2014, MNRAS, 438, 2083

Shahbaz, T., Ringwald, F. A., Bunn, J. C., et al. 1994, MNRAS, 271, L10

Sivakoff, G. R., Tetarenko, A., \& Miller-Jones, J. C. 2015, ATel, 7671, 1

Strong, A. W., Diehl, R., Halloin, H., et al. 2005, A\&A, 444, 495

Terada, K., Miyamoto, S., Kitamoto, S., \& Egoshi, W. 1994, PASJ, 46, 677

Tetarenko, A., Sivakoff, G. R., Gurwell, M. A., et al. 2015a, ATel, 7661, 1

Tetarenko, A., Sivakoff, G. R., Young, K., Wouterloot, J. G. A., \& Miller-Jones, J. C. 2015b, ATel, 7708, 1

Tomsick, J. A., Rahoui, F., Kolehmainen, M., et al. 2015, ApJ, 808, 85

Tsubono, K., Aoki, T., Asuma, K., et al. 2015, ATel, 7701, 1

van der Laan, H. 1966, Nature, 211, 1131

Winkler, C., Courvoisier, T. J.-L., Di Cocco, G., et al. 2003, A\&A, 411, L1

Xie, F.-G., Yang, Q.-X., \& Ma, R. 2014, MNRAS, 442, L110

Życki, P. T., Done, C., \& Smith, D. A. 1999, MNRAS, 309, 561 
Table 1. List of the $>6$ Crab flares and their main properties.

\begin{tabular}{|c|c|c|c|c|c|c|}
\hline Name & $\begin{array}{l}\text { Start }^{a} \\
\text { (MJD) }\end{array}$ & $\begin{array}{l}\text { Peak time } \\
\text { (MJD) }\end{array}$ & $\begin{array}{l}\text { Stop }^{a} \\
\text { (MJD) }\end{array}$ & $\begin{array}{c}\mathrm{CR}_{3-13 \mathrm{keV}^{b}} \\
(\mathrm{cts} / \mathrm{s})\end{array}$ & $\begin{array}{c}\mathrm{CR}_{20-40 \mathrm{keV}^{b}} \\
\quad(\mathrm{cts} / \mathrm{s})\end{array}$ & Properties $^{c}$ \\
\hline $\mathrm{I}$ & 57193.9217 & 57193.9356 & 57193.9402 & 184 & 1215 & Multiple \\
\hline II & 57193.9703 & 57193.9981 & 57194.0827 & 181 & 1234 & Multiple \\
\hline III & 57194.0827 & 57194.1152 & 57194.1428 & 1055 & 5209 & Complex \\
\hline IV & 57194.2232 & 57194.3107 & 57194.3938 & 2010 & 7040 & Isolated/Complex \\
\hline $\mathrm{Va}^{d}$ & 57194.6273 & 57194.6399 & 57194.6521 & $473^{e}$ & 3328 & Multiple/Complex, preceded by plateau \\
\hline $\mathrm{Vb}^{d}$ & 57194.6521 & 57194.6579 & 57194.6708 & 852 & 3999 & Multiple/Complex \\
\hline VI & 57194.6960 & 57194.7346 & 57194.7473 & 129 & 1974 & Isolated/Complex \\
\hline VII & 57194.9788 & 57194.9996 & 57195.0089 & 459 & 2200 & Multiple/Complex \\
\hline VIII & 57195.0089 & 57195.0293 & 57195.0501 & 865 & 4950 & Multiple/Complex \\
\hline IX & 57195.0582 & 57195.0826 & 57195.1095 & 320 & 2386 & Multiple/Complex \\
\hline $\mathrm{X}$ & 57195.2318 & 57195.2503 & $57195.2712^{f}$ & $577^{g}$ & 3472 & Multiple, preceded by succession of $\sim 6$ Crab peaks \\
\hline XI & 57195.4294 & 57195.4388 & 57195.4450 & 857 & 7036 & Multiple \\
\hline $\mathrm{XIIa}^{d}$ & 57195.4450 & 57195.4573 & 57195.4665 & 401 & 3525 & Multiple/Complex \\
\hline $\mathrm{XIIb}^{d}$ & 57195.4665 & 57195.4723 & 57195.4841 & 1231 & 6299 & Multiple, followed by plateau \\
\hline XIII & 57197.1373 & 57197.1785 & 57197.1924 & 2076 & 7081 & Multiple, preceded by plateau \\
\hline XIV & 57197.1924 & 57197.2020 & 57197.2067 & 1240 & 4368 & Multiple \\
\hline $\mathrm{XV}$ & 57197.2124 & 57197.2228 & 57197.2310 & 210 & 1793 & Multiple/Complex \\
\hline XVI & 57197.3450 & 57197.3647 & 57197.3705 & 151 & 1036 & Isolated \\
\hline
\end{tabular}

Notes. MJD 57193 is 2015 June 20. ${ }^{(a)}$ Start (resp. stop) time of a flare is defined as the time $20-40 \mathrm{keV}$ CR reaches $165 \mathrm{cts} / \mathrm{s}$ (1 Crab) during the increase (resp. decrease), or by the minimum reached before (resp. after) the increase (decrease) for multiple flares. The uncertainty on the times is $\pm 6 \times 10^{-4}$ d. ${ }^{(b)}$ Count rates at the peaks. ${ }^{(c)}$ "Multiple" stands for series of well-defined flares occurring in rapid repetition. "Complex" stands for flares showing various peaks. "Plateau" indicates a $>1$ Crab plateau. ${ }^{(d)}$ These peaks appear as single peaks in Fig. 1 . They are in fact true multiples. ${ }^{(e)}$ The $3-13 \mathrm{keV}$ peak time occurred about $200 \mathrm{~s}$ before the $20-40 \mathrm{keV}$ one, indicating a potential hard lag. ${ }^{(f)}$ Data gap at the end of the flare. The stop time is the last point before the gap. ${ }^{(g)}$ The $3-13 \mathrm{keV}$ peak time occurred about $200 \mathrm{~s}$ after the $20-40 \mathrm{keV}$ one, indicating a potential soft lag.

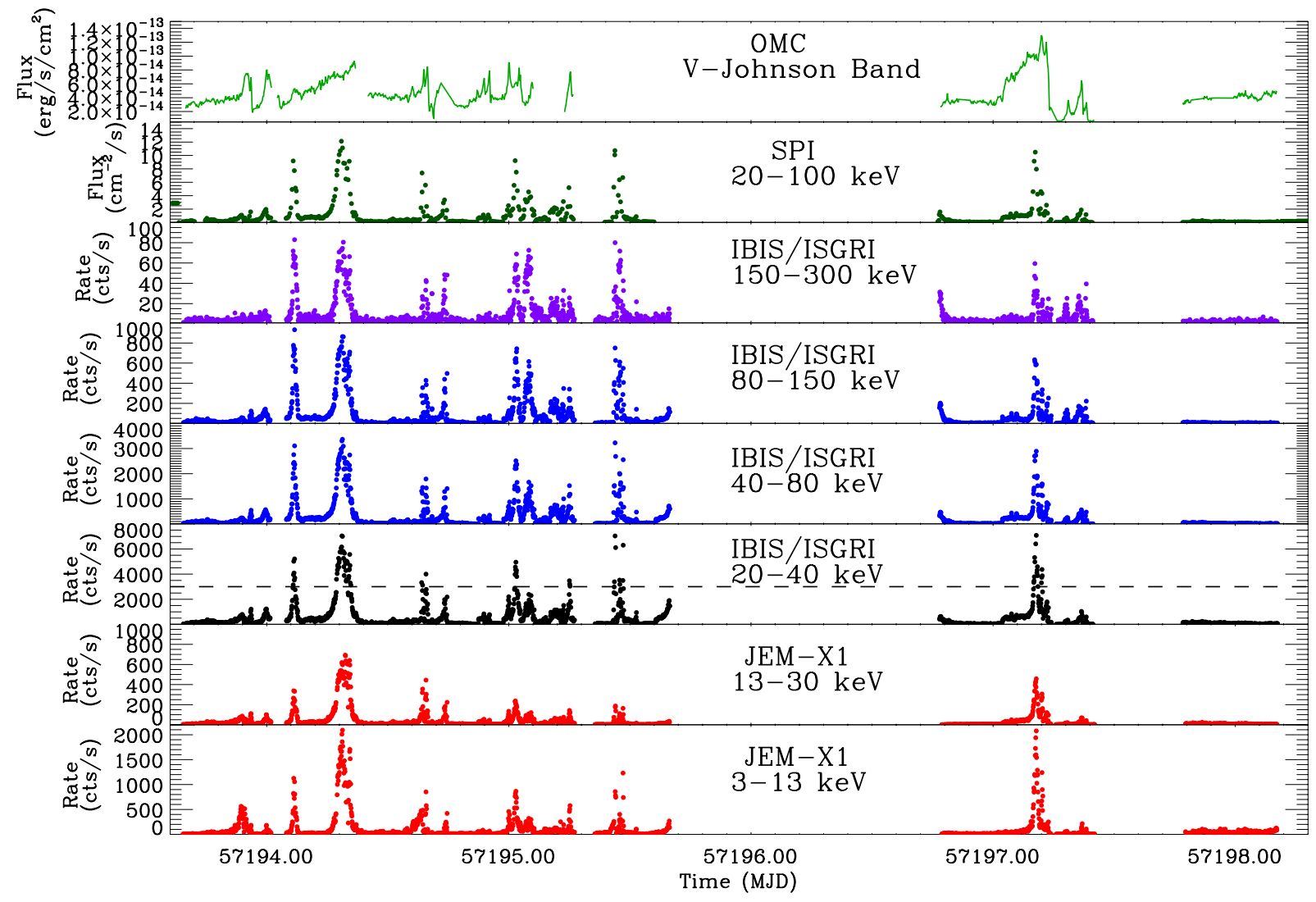

Fig. 4. INTEGRAL LCs of V404 over our 4-day-long observations. All spectral domains considered for the LC extraction are shown here. The dashed line in the 20-40 keV panel represents the approximate level of $L_{\text {Edd }}$ we estimated. MJD 57193 is 2015 June 20. 